\section{DCT winners of BDA}

\section{poster competition}

\section{announced}

The British Dental Association (BDA) have announced the winners of their annual poster competition for dental core trainees (DCTs) in the Hospital Dental Services.

This year, the BDA recognised the excellent and inspiring work of:

- Winning poster: DCTs Emma Elliott, Leeds University Trust, and Alya Omar, St George's Hospital - with Sathyam Sharma DFT - for their poster on 'How effective is workshop teaching at improving dental students' confidence with patient mental health?'

- Runner up: Harriet Moore and Niamh Kelly, DCTs at Ulster Hospital and colleagues for their poster on 'Assessment of facial swellings'.

Two posters were also highly commended:

- Shree Patel and Jaspreet Virdee, the Eastman Dental Hospital, for their poster on 'A WHO surgical safety checklist audit to assess compliance'

- G. Buchanan and K. Gillan, Newcastle upon Tyne Hospitals NHS Trust, for their poster on 'Remote and face-to-face biopsy reviews in OMFS'.

All of the posters can be viewed on the BDA's Facebook page.

Pete Brotherton, Chair of the Hospitals Group, said: 'This was the first year we held this competition entirely online, and it was a great success, though we hope to welcome people back to BDA HQ in the future. Both online and in person, it's always great to get the chance to showcase the work of DCTs from the Hospital Dental Service.

'The winning poster this year was extremely topical with mental health being such an important issue. This poster highlighted the need for undergraduate awareness of the issues of patient mental health. Overall, we were delighted to see the range and quality of the submissions this year from all the specialties and across the UK. Congratulations to all the DCTs for their hard work during the pandemic.'

The winner will receive a $£ 100$ voucher and the runner up $£ 50$, as well as the recognition from their professional associations and their peers.

CONFERENCE REPORT

\title{
The British and Irish Society for Oral Medicine Winter Meeting
}

By Rachel Botrugno, DCT2 in Oral and Maxillofacial Surgery at Addenbrooke's Hospital, Cambridge, UK

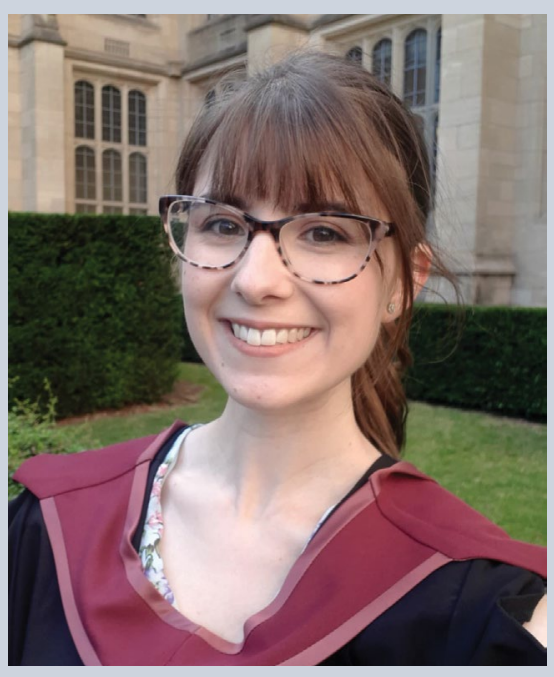

We live in a world in which virtual meetings continue in place of face-toface interaction due to the COVID-19 pandemic. The British and Irish Society for Oral Medicine (BISOM) ran its virtual 2021 Winter Meeting on 18 November with the theme 'Immunobullous diseases'. The Society proposed that delegates would hear from 'expert speakers covering oral, cutaneous, ocular and ENT manifestations, in addition to investigation and management' of these conditions. BISOM certainly achieved this goal through an array of engaging presentations from an expert in each field.

The meeting began with an introduction by the chair Dr Roddy McMillan (BISOM President) and was followed by a morning of five presentations. The afternoon, facilitated by Dr Amanda Willis (Secretary BISOM), started with presentations grouped into original research/quality improvement, followed by oral case reports. These included 'A systematic review of biologic therapies used in the treatment of oral lichen planus'. Clinical case presentations covered interesting conditions such as tuberculosis, syphilis and foregut duplication cyst.

Professor of Oral Dermatology, Jane Setterfield, began the day with 'Oral and cutaneous involvement in immunobullous disease, which provided an excellent overview of these diseases, including prevalence and their clinical presentations.

Dr John Mee, Clinical Scientist at Immunodermatology laboratory, based at St Thomas' Hospital, delivered his talk on 'Immunofluorescence studies in immunobullous disease. This presentation provided delegates with top tips on intraoral biopsies for those patients requiring histopathological investigations for suspected immunobullous diseases, reminding those attending of the science behind direct and indirect immunofluorescence.

'Ocular involvement in immunobullous disease' was the next lecture, delivered by Mr Sajjad Ahmad, Consultant Ophthalmologist. He highlighted the importance of prompt recognition and action for patients at risk of ocular involvement due to the significant sequalae of conjunctival scarring.

Consultant ENT Surgeon Mr Guri Sandhu spoke on the topic of 'Ear, nose and throat involvement in immunobullous disease. His presentation raised awareness of pharyngeal stenosis relating to these conditions demonstrated by endoscopic imaging.

Dr Thomas Tull, Consultant

Dermatologist, provided an interesting talk on the 'Biologics in immunobullous disease'. Rituximab, a human/murine monoclonal antibody, was discussed in detail, touching on its mechanism of action to aid management of immunobullous disease.

The meeting ran very smoothly with easy access to submitted poster presentations allowing delegates to view these in their own time. I want to take this opportunity to thank the BISOM committee for organising this event.

The Society's annual scientific meeting is planned as a face-to-face event in Belfast in spring 2022, which should be another excellent conference for all those involved in or interested in oral medicine. 